\title{
A MORERA TYPE THEOREM IN THE STRIP
}

\section{A. Tumanov}

\section{Introduction}

We prove the following:

Theorem. Let $f$ be a continuous function in the strip $|\operatorname{Im} z| \leq 1$. Suppose for every $r \in \mathbf{R}$ the restriction of $f$ to the circle $|z-r|=1$ extends holomorphically inside the circle. Then $f$ is holomorphic in the strip $|\operatorname{Im} z|<1$.

The result can be regarded as a Morera type theorem because the holomorphic extendibility is equivalent to the moment condition. For various versions of the Morera theorem, see e. g. [ABC], [BZ]. The question answered by the above theorem has been open for over a decade. This is a special case of the general largely unsolved problem whether the analyticity of a function can be tested by restricting the function on (usually one parameter) families of Jordan curves.

Agranovsky and Val'sky [AV] proved the result for any family of curves invariant under Euclidean motions of the plane. Globevnik [G1] proved tests of analyticity in an annulus for any rotation invariant family of Jordan curves. Apparently, the compactness of the group of rotations played an important role, because for the family obtained by translating a given curve parallel to the real line, the problem has since been open even for the circle. Agranovsky [Ag] proved Theorem under additional growth assumptions on $f$. He also proved the result in case $f$ is real analytic in the disc $|z|<1+\epsilon$. Agranovsky and Globevnik [AG] have recently solved the problem for arbitrary one parameter families of circles for rational functions of two real variables and for real analytic functions. In particular, they found families on which the analyticity cannot be tested. Ehrenpreis [Eh] has also proved Theorem for real analytic $f$, but his results hold for PDE more general than the Cauchy-Riemann equation. Globevnik [G2] has recently proved the result for the (two parameter) family of all circles of constant radius $\lambda<2 / 3$ contained in the strip. (For $\lambda<1 / 2$ this follows from [G1].) Finally, Ehrenpreis has informed the author that he has found a different proof of our Theorem above.

Despite the one variable nature of the problem, we use the analysis of several complex variables, specifically, the extendibility of CR functions. Most of the proof consists of references to well-known results. The proof goes through for

Received June 13, 2002. 
a finite strip and some curves other than the circle, but for a general curve the question remains open.

The author thanks Mark Agranovsky for useful discussions.

\section{Proof of the main result}

We prove the theorem stated in the introduction.

Step 1. We introduce a $\mathrm{CR}$ function $F$ on a hypersurface $M \subset \mathbf{C}^{2}$.

Denote by $f_{r}(z)$ the holomorphic extension of $f$ to the disc $|z-r|<1$. Define $F(z, w)=f_{r}(z)$ for $w=z-r$. The function $F$ is defined on the real hyperplane $\operatorname{Im} z=\operatorname{Im} w$ for $|w| \leq 1$ and holomorphic on every disc $z=w+r,|w|<1$, where $r \in \mathbf{R}$ is constant. Hence $F$ is a CR function. (For references on CR functions, see e. g. [BER], [Bo].) On the boundary $E=\{(z, w): \operatorname{Im} z=\operatorname{Im} w,|w|=1\}$ of the region on the hyperplane where $F$ is defined, we have $F(z, w)=f(z)$. Note that $G(w)=-w^{-1}$ reflects the circle $|w|=1$ about the imaginary axis. Then $F(z, G(w))=f(z)$, too. Hence $F$ satisfies $F(z, w)=F(z, G(w))$ on $E$.

Define the hypersurface

$$
M=\{(z, w): \operatorname{Im} z=h(w), w \in \mathbf{C}\},
$$

where $h(w)=\operatorname{Im} w$ for $|w| \leq 1$ and $h(w)=h(G(w))=-\operatorname{Im}\left(w^{-1}\right)$ for $|w| \geq 1$. Then $h$ is continuous and it is smooth except for $|w|=1$. Extend $F$ to the rest of $M$ by $F(z, w)=F(z, G(w))$. Then $F$ is a continuous CR function on $M$.

We will ultimately prove that $F$ extends holomorphically to a neighborhood of $(0,0)$ and that actually $F(z, w)$ is independent of $w$. This will imply that $f_{r}(z)$ is independent of $r$, whence $f$ is holomorphic.

Step 2. We prove that $F$ extends holomorphically to a one-sided neighborhood of each point of $E$ except possibly the points $(z, w) \in E, w= \pm 1$.

Indeed, $M$ consists of two smooth hypersurfaces that meet transversally at the totally real edge $E$ except the points where $w= \pm 1$ in which the two pieces have the same tangent plane (and $E$ has complex tangencies). By the "edge of the wedge" theorem, in a neighborhood of every point $\left(z_{0}, w_{0}\right) \in E$ such that $\pm \operatorname{Im} w_{0}>0, F$ extends holomorphically to the "convex" side of $M$, that is $\pm(\operatorname{Im} z-h(w))<0$ respectively, which completes this step.

Note that by changing the coordinates to $z_{1}=z-w, z_{2}=z+w^{-1}$, we reduce the problem to the "straight" wedge $\pm \operatorname{Im} z_{1}<0, \pm \operatorname{Im} z_{2}<0$. Our conclusion follows e.g. by a version of the "edge of the wedge" theorem for "straight" wedges by Ayrapetian and Henkin [AH]. We however prefer to use $[\mathrm{Ay}]$, where the extension is obtained by means of analytic discs (see also [T1] for a brief proof).

Step 3. We prove that $F$ extends to a (full) neighborhood of every point $(z, w) \in$ $E$ with $w= \pm 1$. This is the only step for which we could not find direct references. We essentially use it for a finite strip only. 
The result is obtained by extending $F$ into small analytic discs. Let $\Delta$ be the standard unit disc in complex plane. Recall an analytic disc in $\mathbf{C}^{2}$ is a map $g: \Delta \rightarrow \mathbf{C}^{2}, g \in C(\bar{\Delta}) \cap \mathcal{O}(\Delta)$. We say the disc $g$ is attached to $M$ if $g(b \Delta) \subset M$. By the Baouendi-Treves [BT] approximation theorem, the CR function $F$ is locally a uniform limit of holomorphic polynomials. (See [T1] for a brief proof of the theorem for CR funtions.) By the maximum principle, the polynomials converge on (the images of) the analytic discs attached to $M$. Hence $F$ extends holomorphically to every open set covered by the analytic discs. (See [BER], [Bo] for details.)

For piecewise smooth hypersurfaces of the form (1) the Baouendi-Treves approximation theorem still holds. Furthermore, since $h$ is globally defined, the approximation theorem holds in the global form, that is if $F$ is a CR function on all of $M$, then the approximations converge to $F$ uniformly on compact sets on $M$. (See $[\mathrm{BD}]$ for a global version of the approximation theorem.) Our situation is particularly simple because the defining function $h$ is independent of Rez. After rescaling $w \rightarrow c w$ with large $c$, the hypersurface $M$ gets "close" to the hyperplane $\operatorname{Im} z=0$ and the construction in the proof of the theorem gives global approximations.

At this point, the reader interested in the proof for the infinite strip $|\operatorname{Im} z| \leq 1$ only can skip to Step 4.

Small analytic discs attached to $M$ of the form (1) are constructed explicitly as follows. Let $\zeta \mapsto g(\zeta)=(z(\zeta)=x(\zeta)+i y(\zeta), w(\zeta)), \zeta \in \Delta$, be an analytic disc attached to $M$. Given the w-component $\zeta \mapsto w(\zeta)$ and $x(0)=x_{0}$, we have $y(\zeta)=h(w(\zeta))$ for $|\zeta|=1$, hence the z-component $\zeta \mapsto z(\zeta)$ is defined as the unique holomorphic function in $\Delta$ with given imaginary part on the circle and given $x(0)$. The "center" $g(0)=\left(x_{0}+i y_{0}, w(0)\right)$ is found by

$$
y_{0}=\frac{1}{2 \pi i} \int_{|\zeta|=1} h(w(\zeta)) \frac{d \zeta}{\zeta} .
$$

We construct the family of $\operatorname{discs} \zeta \mapsto g(\zeta)=g\left(\zeta, x_{0}, w_{0}, t\right)$ for which $w(\zeta)=$ $w_{0}+a \zeta+t \phi(\zeta)$, where $a>0$ is fixed and small enough to make the discs small, and $\phi$ is a fixed holomorphic function with $\phi(0)=0$, which we will choose later. The discs depend on the parameters $x_{0} \in \mathbf{R}, w_{0} \in \mathbf{C}$ close to \pm 1 , and $t \in \mathbf{R}$ close to 0 .

Consider the evaluation map $\Phi:\left(x_{0}, w_{0}, t\right) \mapsto g\left(0, x_{0}, w_{0}, t\right)$. Since $h(\bar{w})=$ $-h(w)$, then (2) yields $y_{0}=0$ for $t=0$. Hence $\Phi\left(x_{0}, \pm 1,0\right)=\left(x_{0}, \pm 1\right)$. We will prove that $\Phi$ is a diffeomorphism in a neighborhood of $\left(x_{0}, \pm 1,0\right)$, so the discs cover a neighborhood of $\left(x_{0}, \pm 1\right)$, which will complete Step 3.

By the implicit function theorem, it suffices to show that $\dot{y}(0)=\left.\frac{d}{d t}\right|_{t=0} y(0) \neq$ 0 for $w_{0}= \pm 1$. Note that $h(w)=\operatorname{Im} H(w)$, where $H(w)=w$ for $|w| \leq 1$ and $H(w)=G(w)$ for $|w|>1$. Then (2) turns into

$$
y(0)=\operatorname{Im}\left(\frac{1}{2 \pi i} \int_{|\zeta|=1} H( \pm 1+a \zeta+t \phi(\zeta)) \frac{d \zeta}{\zeta}\right) .
$$


Differentiating (3) yields

$$
\dot{y}(0)=\operatorname{Im}\left(\frac{1}{2 \pi i} \int_{|\zeta|=1} H^{\prime}( \pm 1+a \zeta) \phi(\zeta) \frac{d \zeta}{\zeta}\right) .
$$

If the above expression vanished for all polynomials $\phi$, then by the moment conditions, $\zeta \mapsto H^{\prime}( \pm 1+a \zeta)$ would extend holomorphically from $b \Delta$ to $\Delta$, which is not the case. Hence $\dot{y}(0) \neq 0$ for some $\phi$, which completes Step 3.

Step 4. We prove that $F$ extends holomorphically to a neighborhood of $M \backslash E$.

Indeed, $M \backslash E$ is a union of complex curves, each of which contains a boundary point of the form $(z, \pm 1) \in E$. By Step $3, F$ is holomorphic at that point, and the conclusion follows by propagation of analyticity along complex curves (Hanges and Treves $[\mathrm{HT}])$. We however do not need the result to the whole strength. As we pointed out in Step 2, the hypersurface $M$ consists of two real hyperplanes $\operatorname{Im} z_{1}=0$ and $\operatorname{Im} z_{2}=0$, and the propagation along each hyperplane follows by the separate analyticity theorem of Bernstein (1912), see e.g. [AR].

We have already mentioned that the result of Step 4 follows directly from Step 2. This is because the one-sided holomorphic extendibility also propagates along complex curves, see e. g. [T2]. Indeed, every (full) complex curve in $M$ has boundary points $(z, w) \in E$ where $\pm \operatorname{Im} w>0$. By Step 2, the function $F$ extends to the side $\pm(\operatorname{Im} z-h(w))<0$ respectively; this property propagates along the curve, and the result follows. In our simplified situation, the propagation follows again by a version of the "edge of the wedge" theorem for "straight" wedges $[\mathrm{AH}]$, but we prefer to use [T2], in which the propagation is obtained by means of analytic discs.

Step 5. We prove that $F(z, w)$ is independent of $w$.

Consider the following one parameter family of discs $D(a), a \geq 1$, with centers at $\left(x_{0}, 0\right)$ for fixed $x_{0} \in \mathbf{R}$ :

$$
D(a)=\left\{(z, w): z=x_{0}+a^{-2} w,|w|<a\right\} .
$$

We now regard the discs $D(a)$ as subsets in $\mathbf{C}^{2}$ rather than mappings of the standard disc. The discs $D(a)$ are attached to $M$.

By the global version of the Baouendi-Treves approximation theorem, there is a sequence of polynomials that converges to $F$ on compact subsets of $M$. This sequence also converges on analytic discs attached to $M$, in particular, on $D(a)$. This sequence also converges in a neighborhood of $(0,0)$ because all the steps 1-4 are done by means of analytic discs.

Using the same notation $F$ for the extension, since $F$ is holomorphic, whence continuous in a neighborhood of $(0,0)$, then $\phi_{a}=\left.F\right|_{D(a)}$ approaches $\phi=\left.F\right|_{z=x_{0}}$ as $a \rightarrow \infty$ in a neighborhood of $(0,0)$. In other words, for small $\left|x_{0}\right|$ and $\epsilon>0$, the function $\phi(w)=F\left(x_{0}, w\right)$ is a uniform limit of $\phi_{a}(w)=F\left(x_{0}+a^{-2} w, w\right)$ in $|w|<\epsilon$ as $a \rightarrow \infty$. 
We claim that $\phi_{a}=\left.F\right|_{D(a)}$ is bounded uniformly in $a$. Indeed, by the construction in Step 1, $\left.F\right|_{b D(a)}$ for $a>1$ is defined using $f_{r}(z)$ with $r=z-G(w)$, where $|w|=a, z=x_{0}+a^{-2} w$. Hence $r=x_{0}+a^{-2} w+w^{-1}=x_{0}+a^{-2}(w+\bar{w})$,

$$
|r| \leq\left|x_{0}\right|+2 a^{-1}
$$

so $r$ whence $f_{r}$ is uniformly bounded in $a$.

Now since $\phi_{a}$ is uniformly bounded in $|w|<a$, then by the Cauchy estimates, $\phi_{a}^{\prime} \rightarrow 0$ as $a \rightarrow \infty$. But since $\phi_{a} \rightarrow \phi$ in $|w|<\epsilon$, then $\phi_{a}^{\prime} \rightarrow \phi^{\prime}$. Hence $\phi^{\prime}=0$ identically, and $\phi$ is constant. Now $F(z, w)$ is independent of $w$ for real $z=x_{0}$, whence for complex $z$. This completes the last step in the proof of the theorem.

Proof for finite strip. Theorem also holds in a finite strip. Indeed, for Steps 1, 2 , and 4 it suffices to assume the hypotheses of the theorem for $r$ in arbitrarily small interval. However, we need to adjust our argument in Step 5 because $F$ is no longer defined on all of $M$ and we cannot use the global approximation theorem. Instead, we use the classical Hartogs continuity principle. For small $\epsilon>0$ and $1 \leq a \leq 2$, consider

$$
D(a, \epsilon)=\left\{(z, w): z=x_{0}+a^{-2} w,|w|<a-\epsilon\right\}
$$

By the above construction including Step 3, there is $\epsilon>0$ such that the extension of $F$ is holomorphic on the boundaries of the discs $D(a, \epsilon)$ for all $1 \leq a \leq 2$. (This is not the case for $D(1)$, which is why we have to shrink the discs $D(a)$ for $a$ close to 1 . To complete Step 3 for the points $z=w= \pm 1$, we need $f_{r}$ for all $r$ close to 0 and \pm 2 respectively.)

Now $F$ is holomorphic in a neighborhood of the whole disc $D(1, \epsilon)$ and on the boundaries of the discs $D(a, \epsilon), 1 \leq a \leq 2$. By the continuity principle, $F$ extends along the discs $D(a, \epsilon), 1 \leq a \leq 2$, and then along the discs $D(a), a \geq 2$. The analytic continuations of $F$ along all the discs match because they have the same center. Then we pass to the limit as $a \rightarrow \infty$ and obtain the result as above.

Note that since we use the $\operatorname{discs} D(a)$ for all $a>1$, then by (4) it follows that we need the hypotheses of the theorem for all $|r|<2+\epsilon$ for some $\epsilon>0$. On the other hand we don't need all the values of $f_{r}(z)$ for those $r$-s, but only for $|z|<1+\epsilon,|\operatorname{Im} z| \leq 1$. It would be interesting to find out whether these estimates are sharp.

\section{Proof for other curves}

Our method applies to some curves other than the circle.

Theorem'. Let $a>0, b>0$. Let $f$ be a continuous function in the strip $|\operatorname{Im} z| \leq b$. Suppose that for every $r \in \mathbf{R}$ the restriction of $f$ to the ellipse 
$a^{-2}(x-r)^{2}+b^{-2} y^{2}=1$, where $z=x+i y$, extends holomorphically inside the ellipse. Then $f$ is holomorphic in the strip $|\operatorname{Im} z|<b$.

Proof. The proof consists of the same five steps. We give a proof in the case $a<b$, the other case being similar, even simpler. We normalize $a$ and $b$ so that $b^{2}-a^{2}=1$, then we fix a number $p>0$, such that $a=\sinh p, b=\cosh p$. Denote by $E_{q}$ the ellipse with semi-axes $(\sinh q, \cosh q)$ centered at the origin and by $D_{q}$ the domain bounded by $E_{q}$.

Following Step 1, we introduce $F(z, w)=f_{r}(z)$ for $w=z-r$, where $f_{r}(z)$ is the extension of $f$ into $D_{p}+r, r \in \mathbf{R}$. Then $F$ is a CR function on the hyperplane $\operatorname{Im} z=\operatorname{Im} w, w \in D_{p}$.

For $q \in \mathbf{R}$, we introduce the function

$$
G_{q}(w)=(\cosh q) w+(\sinh q) \sqrt{w^{2}+1}
$$

holomorphic in the exterior of the line segment $[-i, i]$, where we choose the branch of the square root such that $\sqrt{w^{2}+1}>0$ for $w>0$. Note $G_{q}$ is an odd function.

For $q, r, t \in \mathbf{R}, w=\sinh (q+i t)=\sinh q \cos t+i \cosh q \sin t \in E_{q}$, we have $G_{r}(w)=\sinh ( \pm r+q+i t)$ if $\pm q>0$ respectively. Hence $G_{r}\left(E_{q}\right)=E_{|r+q|}$ for $q>0$. From the above properties it follows that $G_{-2 p}$ gives a conformal mapping of $D_{2 p} \backslash[-i, i]$ to itself, and reflects $E_{p}$ about the imaginary axis.

We define $M$ by the equation $\operatorname{Im} z=h(w)$, where $h(w)=\operatorname{Im} w$ for $w \in D_{p}$, and extends to the whole plane applying the relation $h(w)=h\left(G_{-2 p}(w)\right)$ infinitely many times. We extend $F$ to the rest of $M$ so that $F(z, w)=F\left(z, G_{-2 p}(w)\right)$ is also applied infinitely many times.

This completes Step 1 of the proof. Steps $2-4$ go through along the same lines.

In Step 5 we consider the $\operatorname{discs} D(n)$ given by $z=x_{0}+\frac{w}{\cosh (2 n p)}, w \in D_{2 n p}$, where $n$ is a positive integer. Then for small $w$, the function $\phi(w)=F\left(x_{0}, w\right)$ is approximated by $\phi_{n}(w)=F\left(x_{0}+\frac{w}{\cosh (2 n p)}, w\right)$ as $n \rightarrow \infty$. The function $\phi_{n}$ is defined in $D_{2 n p}$ and uniformly bounded in $n$. Hence, $\phi$ is constant, and the proof is complete.

We consider one more example of a curve for which our method works. This may be of interest because the curve meets the boundaries of the horizontal strip at a nonzero angle. Let $\Gamma=\Gamma_{+} \cup \Gamma_{-}$, where $\Gamma_{ \pm}$are the circular arcs defined as follows

$$
\Gamma_{ \pm}=\{z:|z \sin \alpha \pm \cos \alpha|=1, \pm \operatorname{Re} z \geq 0\} .
$$

The arcs intersect at the angle $2 \alpha$, where we choose $\alpha=\pi / n, n \geq 3$ is integer. The proof is similar. The defining function $h$ of the hypersurface $M$ is constructed using the relation $h(w)=h(G(w))$, where $G(w)=(w+$ $\tan \alpha)(-w \tan \alpha+1)^{-1} \operatorname{maps} \Gamma_{-}$to $\Gamma_{+}$. 


\section{References}

[Ag] M. Agranovsky, Private communication.

[AR] N. I. Akhiezer and L. I. Ronkin, On separately analytic functions of several variables and theorems on the "thin end of the wedge", Uspekhi Mat. Nauk 28 (1973), 27-42.

[Ay] R. A. Ayrapetian, Continuation of CR-functions from piecewise-smooth CR-manifolds, Mat. Sb. (N.S.) 134(176) (1987), 108-118, 143.

[AG] M. Agranovsky and J. Globevnik, Analyticity on circles for rational and real analytic functions of two real variables, Preprint series, Institute of Math., Phys., and Mech., University of Ljubljana, vol. 39 (2001), 756, 1-43.

[ABC] M. Agranovsky, C. Berenstein, and D.-C. Chang, Morera theorem for holomorphic $H^{p}$ spaces in the Heisenberg group, J. Reine und Angew. Math. 443 (1993), 49-89.

[AV] M. Agranovsky and R. Val'sky, Maximality of invariant algebras of functions, Siberian Math. J. 12 (1971), 1-7.

[AH] R. A. Ayrapetian and G. M. Henkin, Analytic continuation of CR functions through the "edge of the wedge". Dokl. Akad. Nauk SSSR 259 (1981), 777-781.

[BER] M. S. Baouendi, P. Ebenfelt, and L. P. Rothschild, Real Submanifolds in Complex Space and their Mappings, Princeton Math. Series 47, Princeton Univ. Press, 1999.

[BT] M. S. Baouendi and F. Treves, A property of the functions and distributions annihilated by a locally integrable system of complex vector fields, Ann. of Math. 114 (1981), 387-421.

[BZ] C. Berenstein and L. Zalcman, Pompeiu's problem on symmetric spaces, Comm. Math. Helv. 55 (1980), 593-621.

[Bo] A. Boggess, CR manifolds and the tangential Cauchy-Riemann complex, CRC Press, 1991.

[BD] A. Boggess and R. Dwilewicz, CR Approximation on a Non-rigid Hypersurface Graph in $\mathbf{C}^{n}$, to appear.

[Eh] L. Ehrenpreis, Three problems at Mount Holyoke, Contemp. Math., 278, Providence, RI, 2001.

[G1] J. Globevnik, Testing analyticity on rotation invariant families of curves, Trans. Amer. Math. Soc. 306 (1988), 401-410.

[G2] Holomorphic extensions from open families of circles, Trans. Amer. Math. Soc. 355 (2003), 1921-1931

[HT] N. Hanges and F. Treves, Propagation of holomorphic extendibility of CR function, Math. Ann. 263 (1983), 157-177.

[T1] A. Tumanov, Analytic discs and the extendibility of CR functions. Integral geometry, Radon transforms and complex analysis (Venice, 1996), 123-141, Lecture Notes in Math., 1684, Springer, Berlin, 1998.

[T2] Connections and propagation of analyticity for CR functions, Duke Math. J. 73 (1994), no. $1,1-24$.

Department of Mathematics, University of Illinois, Urbana, IL 61801.

E-mail address: tumanov@math.uiuc.edu 